February 1998

SNUTP 98-005

\title{
Integrable Extension of Nonlinear Sigma Model
}

\author{
Phillial $\mathrm{Oh}^{1}$ \\ Department of Physics, Sung Kyun Kwan University, Suwon 440-746, Korea
}

\begin{abstract}
We propose an integrable extension of nonlinear sigma model on the target space of Hermitian symmetric space (HSS). Starting from a discussion of soliton solutions of $O(3)$ model and an integrally extended version of it, we construct general theory defined on arbitrary HSS by using the coadjoint orbit method. It is based on the exploitation of a covariantized canonical structure on HSS. This term results in an additional first-order derivative term in the equation of motion, which accommodates the zero curvature representation. Infinite conservation laws of nonlocal charges in this model are derived.
\end{abstract}

PACS codes: 11.10.Lm, 11.10.Kk

${ }^{1}$ E-mail: ploh@newton.skku.ac.kr 
The study of integrability of the nonlinear sigma model (NLSM) on the target space of homogeneous space $G / H$ was initiated in the work of [1] where it was discovered that $O(N)$ NLSM admits a zero curvature representation, and infinite family of local and nonlocal conserved charges [2] was derived along with one parameter family of Bäcklund transformation. This result was immediately generalized to the target space of $C P(N)$ [3] and to the principal chiral $S U(N)$ model where nontrivial soliton solutions were obtained by applying the inverse scattering technique 四. Later, the complete integrability on the target space of Riemannian symmetric spaces was established through the works of [5], and various aspects of integrability were developed and applied to the survey of two dimensional quantum field theory [6].

In this Letter, we search for a possible integrable extension of the NLSM on the homogeneous space $G / H$. Our main result is that the NLSM allows an integrable extension when the target space is given by the Hermitian symmetric space (HSS) which is a symmetric space equipped with a complex structure acting on the coset [7]. In achieving this, a covariantized canonical structure term on HSS is added to the original action which results in a first-order total derivative in the equation of motion, and this is found to be completely integrable. In the process, we use the coadjoint orbit method [ [ as an essential tool. We start from a discussion of an integrable extension of the simple $O(3)$ model in this method and its soliton solution to give some motivation.

Let us consider the action of $O(3)$ NLSM:

$$
S=\frac{1}{2} \int d^{2} x \partial_{\mu} \vec{Q} \cdot \partial^{\mu} \vec{Q}, \quad \vec{Q} \cdot \vec{Q}=1 .
$$

We consider solution of the following equation; ( with prime $=\frac{\partial}{\partial x}$, dot $=\frac{\partial}{\partial t}$ )

$$
\vec{Q} \times \vec{Q}^{\prime \prime}-\vec{Q} \times \ddot{\vec{Q}}=0
$$


Note that cross product with $\vec{Q}$ reproduces the formal equation of motion of (1), $\square \vec{Q}-(\vec{Q} \cdot \square \vec{Q}) \vec{Q}=0$. We use the standard parameterization for $S^{2}$ given by $\vec{Q}=(\sin \theta \cos \phi, \sin \theta \sin \phi, \cos \theta)$. Substitution into (2) leads to

$$
\begin{aligned}
2 \cos \theta \theta^{\prime} \phi^{\prime}+\sin \theta \phi^{\prime \prime}-2 \cos \theta \dot{\theta} \dot{\phi}-\sin \theta \ddot{\phi} & =0, \\
\theta^{\prime \prime}-\sin \theta \cos \theta \phi^{\prime 2}-\ddot{\theta}+\sin \theta \cos \theta \dot{\phi}^{2} & =0 .
\end{aligned}
$$

Let us try solutions of the form $\theta \equiv \theta(x-v t), \phi \equiv \hat{\phi}(x-v t)+\Omega t$. We obtain

$$
\begin{aligned}
& 0=2\left(1-v^{2}\right) \cos \theta \theta_{\xi} \hat{\phi}_{\xi}+\left(1-v^{2}\right) \sin \theta \hat{\phi}_{\xi \xi}+2 \Omega v \cos \theta \theta_{\xi} \\
& 0=\left(1-v^{2}\right) \theta_{\xi \xi}-\left(1-v^{2}\right) \sin \theta \cos \theta \hat{\phi}_{\xi}^{2}-2 v \Omega \sin \theta \cos \theta \hat{\phi}_{\xi}+\Omega^{2} \sin \theta \cos \theta,
\end{aligned}
$$

with $\xi=x-v t$. Let $\chi=\left(1-v^{2}\right) \sin \theta \hat{\phi}_{\xi}(|v|<1)$. Then, from (5)

$$
\chi_{\xi}=-\theta_{\xi}(2 v \Omega \cos \theta+\chi \cot \theta)
$$

which upon integration yields

$$
\chi=\frac{v \Omega\left(\cos 2 \theta+c_{0}\right)}{2 \sin \theta}
$$

From (6), we obtain

$$
\theta_{\xi}^{2}+\frac{\chi^{2}}{\left(1-v^{2}\right)^{2}}-\frac{\Omega^{2} \cos 2 \theta}{2\left(1-v^{2}\right)}=E
$$

We choose $c_{0}=-1$, which simplifies $\chi$ to $\chi=-v \Omega \sin \theta$.

Let us consider the effective potential $\theta_{\xi}^{2}+V_{\text {eff }}(\theta)=E$ given by

$$
V_{e f f}(\theta)=-\frac{\Omega^{2} \cos ^{2} \theta}{\left(1-v^{2}\right)^{2}}+\frac{\Omega^{2}\left(1+v^{2}\right)}{2\left(1-v^{2}\right)} .
$$

For a soliton solution, we choose $E=\frac{\Omega^{2}\left(1+v^{2}\right)}{2\left(1-v^{2}\right)}$, which yields

$$
\theta_{\xi}^{2}=\frac{\Omega^{2}}{\left(1-v^{2}\right)^{2}} \cos ^{2} \theta .
$$


Note that differentiation of the above equation with respect to $\xi$ leads to the sineGordon equation in terms of $\bar{\theta} \equiv 2 \theta$ [1]. One can show that the solution is given by

$$
\sin \theta= \pm \tanh \beta\left(\xi-\xi_{0}\right)
$$

with $\beta=\frac{\Omega}{1-v^{2}}$. Hence we obtain

$$
\begin{aligned}
\theta(x, t) & =\sin ^{-1}\left[ \pm \tanh \left(\frac{\Omega}{1-v^{2}}\left(x-v t-\xi_{0}\right)\right)\right] \\
\phi(x, t) & =\phi_{0}-\frac{v \Omega}{1-v^{2}}(x-v t)+\Omega t
\end{aligned}
$$

We find that $\Omega=0$ produces only trivial solution, and $v=0$ gives the stationary soliton solution. The soliton is of topological nature, because $\sin \theta \rightarrow \mp 1$, as $x \rightarrow \mp \infty$ or $x \rightarrow \pm \infty[9]$.

Motivated by the above procedure, we search for a simple possible integrable extension in the form

$$
\vec{Q} \times \ddot{\vec{Q}}-\vec{Q} \times \vec{Q}^{\prime \prime}=\gamma^{0} \dot{\vec{Q}}+\gamma^{1} \vec{Q}^{\prime}
$$

where $\gamma^{0}$ and $\gamma^{1}$ are constants. This form is suggested by an observation that with $\gamma^{1}=0$ and in absence of second order time derivative term in the left hand side, the above is the continuous Heisenberg ferromagnet equation which is well-known to be completely integrable [10]. In terms of $\xi=x-v t$, the above equation has a good chance of being integrable.

Again let us use $\vec{Q}=(\sin \theta \cos \phi, \sin \theta \sin \phi, \cos \theta)$ and try solution of the form $\theta \equiv \theta(\xi), \phi \equiv \hat{\phi}(\xi)+\Omega t$. Substitution into (15) gives

$$
\begin{aligned}
-v \gamma^{0} \theta_{\xi}+\gamma^{1} \theta_{\xi} & =2\left(1-v^{2}\right) \cos \theta \theta_{\xi} \hat{\phi}_{\xi}+\left(1-v^{2}\right) \sin \theta \hat{\phi}_{\xi \xi}+2 v \Omega \cos \theta \theta_{\xi} \\
v \gamma^{0} \sin \theta \hat{\phi}_{\xi} & -\gamma^{0} \Omega \sin \theta-\gamma^{1} \sin \theta \hat{\phi}_{\xi}=\Omega^{2} \sin \theta \cos \theta \\
& +\left(1-v^{2}\right) \theta_{\xi \xi}-\left(1-v^{2}\right) \sin \theta \cos \theta \hat{\phi}_{\xi}^{2}-2 v \Omega \sin \theta \cos \theta \hat{\phi}_{\xi} \cdot(
\end{aligned}
$$


Let $\chi=\left(1-v^{2}\right) \sin \theta \hat{\phi}_{\xi}(|v|<1)$ as before. Then, from (16) we find

$$
\chi=\frac{v \Omega \cos 2 \theta-2 \Gamma \cos \theta+c}{2 \sin \theta}
$$

with $\Gamma=\gamma^{1}-v \gamma^{0}$. (17) gives

$$
E=\theta_{\xi}^{2}+\frac{\chi^{2}}{\left(1-v^{2}\right)^{2}}-\frac{\Omega^{2} \cos 2 \theta}{2\left(1-v^{2}\right)}-\frac{2 \Omega \gamma^{0} \cos \theta}{\left(1-v^{2}\right)} .
$$

With $\Gamma=2 \Omega v, c=3 \Omega v$, we have

$$
\chi=\frac{\Omega v(\cos \theta-1)^{2}}{\sin \theta} .
$$

And the effective potential is given by (19) as

$$
V_{e f f}(\theta)=\frac{\Omega^{2} v^{2}}{\left(1-v^{2}\right)^{2}} \frac{(1-\cos \theta)^{2}}{1+\cos \theta}\left[(1-\cos \theta)-\frac{1-v^{2}}{v^{2}}(1+\cos \theta)\right]+\frac{3 \Omega^{2}}{2\left(1-v^{2}\right)},
$$

where we have set $\gamma^{0}=-\Omega$. It can be put into the following expression;

$$
V(\theta)=\frac{4 \Omega^{2}}{\left(1-v^{2}\right)^{2}} \frac{\sin ^{4} \frac{\theta}{2}}{\cos ^{2} \frac{\theta}{2}}\left[\sin ^{2} \frac{\theta}{2}-\left(1-v^{2}\right)\right]+\frac{3 \Omega^{2}}{2\left(1-v^{2}\right)} .
$$

Defining $\beta \equiv \frac{\Omega}{1-v^{2}}, \alpha^{2} \equiv\left(1-v^{2}\right)$ and choosing $E=\frac{3 \Omega^{2}}{2\left(1-v^{2}\right)}$, we find

$$
\theta_{\xi}^{2}+4 \beta^{2} \frac{\sin ^{4} \frac{\theta}{2}}{\cos ^{2} \frac{\theta}{2}}\left(\sin ^{2} \frac{\theta}{2}-\alpha^{2}\right)=0
$$

Let $\omega=\sin \frac{\theta}{2}$. Then, (23) becomes

$$
d \omega=\mp \beta \omega^{2} \sqrt{\alpha^{2}-\omega^{2}} d \xi,
$$

which can be immediately integrated to yield

$$
\omega= \pm \frac{\alpha}{\sqrt{1+\alpha^{4} \beta^{2}\left(\xi-\xi_{0}\right)^{2}}} .
$$

Hence the soliton solution is given by

$$
\theta(x, t)=2 \sin ^{-1}\left[ \pm \sqrt{\frac{1-v^{2}}{1+\Omega^{2}\left(x-v t-\xi_{0}\right)^{2}}}\right] .
$$


Also from $\chi=\left(1-v^{2}\right) \sin \theta \hat{\phi}_{\xi}=\Omega v \frac{(1-\cos \theta)^{2}}{\sin \theta}$, we have

$$
\phi(x, t)=\phi_{0}+\tan ^{-1}\left[\left(\frac{\Omega}{v}\right)\left(x-v t-\xi_{0}\right)\right]+\Omega t .
$$

Unlike the previous case, this soliton is non-topological: $\sin (\theta / 2)$ starts at 0 at $\xi=-\infty$, reaches $\pm \sqrt{1-v^{2}}$ at $\xi=\xi_{0}$, and goes back to 0 at $\xi=\infty$.

The above analysis suggests that the first order derivative term in the right hand side of (15) could be one possible integrable extension of $O(3)$ model. It originates from the covariantized symplectic structure on $S^{2}$ and can be generalized to arbitrary HSS, as can be seen from the canonical structure of integrable generalized Heisenberg ferromagnet system on HSS [8]. To describe a general formalism on HSS, we start with a brief summary of the coadjoint orbit approach to NLSM [8]. Consider a group $G$, Lie algebra $\mathcal{G}$ and its dual $\mathcal{G}^{*}: X \in \mathcal{G} ; u \in \mathcal{G}^{*}$. Let us assume that the inner product is given by the trace: $\langle u, X>=\operatorname{Tr}(X u)$. Then, $\mathcal{G}$ and $\mathcal{G}^{*}$ are isomorphic and the coadjoint orbit, which is generated as the orbit of coadjoint action of the group $G$ can be parametrized by

$$
Q=g K g^{-1}=Q^{A} t^{A} ; K \in \mathcal{G}(A=1, \cdots, \operatorname{dim} \mathcal{G})
$$

where $t^{A}$ 's are the generators of $\mathcal{G}$. One can see that $Q$ on each coadjoint orbit characterized by the element $K$ is defined on a homogeneous space $G / H$, where $H$ is the stabilizer of the point of $K$. It is well-known that there is a natural symplectic structure on each orbit, which comes from the cotangent bundle $T^{*} G \cong G \times \mathcal{G}^{*}$ via symplectic reduction [1]. The canonical one-form on $G / H$ which has a left global $G$ and a right local $H$ invariance is given by

$$
\Theta=<\operatorname{Ad}^{*}(g) K, \delta g g^{-1}>=\operatorname{Tr}\left(K g^{-1} \delta g\right)
$$

Based on the above, we propose the following action for the NLSM on the 
target space of coadjoint orbit:

$$
S=\int d^{2} x \operatorname{Tr}\left[\partial_{\mu} Q \partial^{\mu} Q+2 \gamma^{\mu}\left(K g^{-1} \partial_{\mu} g\right)\right]
$$

where $\gamma^{\mu}$ is a two-vector. Note that the above action has a right local $H$ symmetry which is responsible for the reduction to the coset space $G / H$. The first term becomes the standard Lagrangian of NLSM on $G / H$ [8]; $\sim g_{\alpha \beta} \partial_{\mu} \bar{\psi}^{\alpha} \partial^{\mu} \psi^{\beta}$, where $g_{\alpha \beta}$ is the metric in terms of the local coordinate $\psi^{\alpha}$ on $G / H$. The second term is the covariantized canonical structure of (29) on the coadjoint orbit. In a frame with $\gamma^{0}=1$ and $\gamma^{1}=0$, it becomes the canonical structure $\sim p \dot{q}$. The equation of motion with respect to the variation of $g$ is given by

$$
\partial_{\mu}\left[Q, \partial^{\mu} Q\right]+\gamma^{\mu} \partial_{\mu} Q=0
$$

Note that in $S U(2)$ case with $Q=\vec{Q} \cdot \vec{t}$, the above equation reduces to (15).

To demonstrate a complete integrability of (31) on HSS [7], we first explain HSS in terms of coadjoint orbit language. Let us recall that symmetric space is a coset space $G / H$ for Lie groups whose associated Lie algebras $\mathcal{G}$ and $\mathcal{H}$, with the decomposition $\mathcal{G}=\mathcal{H} \oplus \mathcal{M}$, satisfy the commutation relations,

$$
[\mathcal{H}, \mathcal{H}] \subset \mathcal{H}, \quad[\mathcal{H}, \mathcal{M}] \subset \mathcal{M}, \quad[\mathcal{M}, \mathcal{M}] \subset \mathcal{H}
$$

For HSS, (i) the element $K$ is chosen as the central element of the Cartan subalgebra of $\mathcal{G}$ whose centralizer in $\mathcal{G}$ is $H$. (ii) Then, we have $J=\operatorname{Ad}(K)$ acting on the coset is a linear map satisfying the complex structure condition $J^{2}=-1$, which along with (32) gives the identity [8]:

$$
\left[Q,\left[Q, \partial_{\mu} Q\right]\right]=-\partial_{\mu} Q
$$

Next, we give the zero curvature representation of (31). Introduction of

$$
A_{\mu}=a\left[Q, \partial_{\mu} Q\right]+b \epsilon_{\mu}^{\rho}\left[Q, \partial_{\rho} Q\right]+c_{\mu} Q
$$


and substitution into

$$
\epsilon^{\mu \nu}\left(\partial_{\mu} A_{\nu}-\partial_{\nu} A_{\mu}+\left[A_{\mu}, A_{\nu}\right]\right)=0
$$

with use of (33) for HSS yield

$$
\begin{aligned}
0= & 2 b \partial_{\mu}\left[Q, \partial^{\mu} Q\right]+2 \epsilon^{\mu \nu} c_{\nu} \partial_{\mu} Q+2 a \epsilon^{\mu \nu} c_{\nu} \partial_{\mu} Q-2 b c^{\mu} \partial_{\mu} Q \\
& +\left(a^{2}+2 a-b^{2}\right) \epsilon^{\mu \nu}\left[\partial_{\mu} Q, \partial_{\nu} Q\right] .
\end{aligned}
$$

We have ( with $\epsilon^{01}=1, \eta_{\mu \nu}=(-,+), \epsilon^{\mu \nu} \epsilon_{\nu}{ }^{\sigma}=\eta^{\mu \sigma}$ )

$$
a^{2}+2 a-b^{2}=0, \epsilon^{\mu \nu}(1+a) c_{\nu}-b c^{\mu}=b \gamma^{\mu} .
$$

Writing $(a+1)^{2}-b^{2}=1$, we have

$$
a=\frac{2}{\lambda^{2}-1}, \quad b=\frac{2 \lambda}{\lambda^{2}-1} .
$$

From the second equation of (37), we find

$$
c_{\mu}=\frac{4 \lambda^{2}}{\left(\lambda^{2}-1\right)^{2}} \gamma_{\mu}+\frac{2 \lambda\left(\lambda^{2}+1\right)}{\left(\lambda^{2}-1\right)^{2}} \epsilon_{\mu}^{\nu} \gamma_{\nu}
$$

Note that in the case of $c^{\mu}=\gamma^{\mu}=0$, the condition (37) is precisely the one emerging from the zero curvature condition of NLSM on the homogeneous symmetric target space [5].

Permitting the zero curvature condition, (31) has an infinite number of local [10] and nonlocal [2] conservation laws. We compute the nonlocal conserved charge here by making use of the well-known technique from the analysis of the principal chiral model [12, 13, 14]. Given the linear problem,

$$
\left(\partial_{\mu}+A_{\mu}\right) \psi(x ; \lambda)=0
$$

let us consider the Laurent expansion around $\frac{1}{\lambda}$,

$$
\psi(x ; \lambda)=\sum_{n=0}^{\infty} \frac{\psi_{n}}{\lambda^{n}}, \quad \psi_{0}=1 .
$$


The infinitely many conserved currents are defined as

$$
J_{\mu}^{(n)}=\epsilon_{\mu \nu} \partial^{\nu} \psi_{n}(x)
$$

which automatically satisfies the current conservation $\partial_{\mu} J^{\mu}=0$. The $\psi_{n}$ 's are determined recursively as follows: Substituting (34), (38), and (39) into (40), one obtains $\left(j_{\mu} \equiv 2\left[Q, \partial_{\mu} Q\right]\right)$ in the lowest order in $\frac{1}{\lambda}$

$$
\partial_{\mu} \psi_{1}+\epsilon_{\mu \nu}\left(j^{\nu}+2 \gamma^{\nu} Q\right)=0
$$

whose current conservation is nothing but the equation of motion (31). The next lowest order produces

$$
\partial_{\mu} \psi_{2}+\left(j_{\mu}+4 \gamma_{\mu} Q\right)+\epsilon_{\mu \nu}\left(j^{\nu}+2 \gamma^{\nu} Q\right) \psi_{1}=0
$$

The higher order yields the following relations $(p \geq 0)$;

$$
\begin{aligned}
0 & =\partial_{\mu} \psi_{p+3}+\epsilon_{\mu \nu} \sum_{m=0}^{[p / 2]+1}\left(j^{\nu}+2(m+1) \gamma^{\nu} Q\right) \psi_{p+2-2 m} \\
& +\sum_{m=0}^{\left[\frac{p+1}{2}\right]}\left(j_{\mu}+4(m+1) \gamma_{\mu} Q\right) \psi_{p+1-2 m}+2 \epsilon_{\mu \nu} \gamma^{\nu} Q \sum_{m=0}^{[p / 2]}(m+1) \psi_{p-2 m}
\end{aligned}
$$

which determines $\psi_{n}(n \geq 3)$ completely in terms of lower $\psi_{n}$ 's.

The conserved charges can be constructed in two separate cases depending on the boundary conditions. One is the periodic boundary condition $Q(x+2 L)=$ $Q(x)$. The conserved charges are given by $M^{(n)}=\int_{-L}^{+L} J_{0}^{(n)}=\psi(-L)-\psi(+L)$. (43) yields

$$
M^{(1)}(Q)=\int_{-L}^{+L} d x\left(j^{0}(x)+2 \gamma^{0} Q(x)\right) .
$$

The next order (44) produces the nonlocal conserved charge as

$$
\begin{aligned}
M^{(2)}(Q) & =\int_{-L}^{+L} d x\left(j^{1}(x)+4 \gamma^{1} Q(x)\right) \\
& +\int_{-L}^{+L} d x \int_{-L}^{+L} d x^{\prime} \theta\left(x-x^{\prime}\right)\left(j^{0}(x)+2 \gamma^{0} Q(x)\right)\left(j^{0}\left(x^{\prime}\right)+2 \gamma^{0} Q\left(x^{\prime}\right)\right) .
\end{aligned}
$$


The expressions for higher conserved charges follow from (45). In the rapidly decreasing case [10] with the boundary condition given by $\lim _{|x| \rightarrow \infty} Q(x)=Q_{0}(\neq 0)$, the above integrals with $\pm L$ being replaced by $\pm \infty$ diverge in general. They become finite after subtracting their values in the ground state, i.e., $M_{r e g}^{(n)}=$ $M^{(n)}(Q)-M^{(n)}\left(Q_{0}\right)$.

In summary, starting from a discussion of soliton solutions of simple $O(3)$ NLSM and an integrally extended version of it, we verified that NLSM with the target space of arbitrary HSS allows an integrable extension by using the coadjoint orbit formulation. The covariant symplectic structure term on HSS results in the completely integrable equation of motion, and an explicit expression for infinite number of nonlocal currents was obtained. A more detailed analysis on the subject of classical and quantum integrability such as Hamiltonian formulation and Poisson structure, current algebra, and multi-soliton solutions will be addressed elsewhere.

I thank Q-H. Park for useful discussions. This work is supported in part by the Korea Science and Engineering Foundation through the Center for Theoretical Physics at Seoul National University, and the project number (95-0702-04-01-3), and by the Ministry of Education through the Research Institute for Basic Science (BSRI/97-1419).

\section{References}

[1] Pohlmeyer K 1976 Commun. Math. Phys. 46207

[2] Lüsher M and Pohlmeyer K 1978 Nucl. Phys. B 13746

[3] Eichenherr H 1978 Nucl. Phys. B 146215

[4] Zakharov V E and Mikhailov A V 1978 JETP Lett. 2742 
[5] Eichenherr H and Forger M 1979 Nucl. Phys. B 155 381, ibid 1980164528 , and Commun. Math. Phys. 82227

[6] Abdalla E, Abdalla M C B and Rothe K D 1991, 2 Dimensional Quantum Field Theory (World Scientific, Singapore)

[7] Helgason S 1978 Differential Geometry, Lie Groups, and Symmetric Spaces (Academic Press, New York); Fordy A P and Kulish P P 1983 Commum. Math. Phys. 89 427; Perelomov A M 1987 Phys. Rep. 146135

[8] Oh P and Park Q-H 1996 Phys. Lett. B 383333

[9] Since our $\theta$ is defined to be $0 \leq \theta \leq \pi, \xi$ must be restricted to be in the range $\xi_{0} \leq \xi<\infty$ in the solution (12).

[10] Faddeev L D and Takhtajan L A 1987 Hamiltonian Methods in the Theory of Solitons (Springer-Verlag, Berlin)

[11] Abraham R and Marsden J E 1978 Foundations of Mechanics (Addison Wesley, New York)

[12] de Vega H J 1979 Phys. Lett. B 87233

[13] Brézin E, Itzykson C, Zinn-Justin J and Zuber J-B 1979 Phys. Lett. B 82442

[14] See also Ogielski A T 1980 Phys. Rev. D 21 406; Ogielski A T, Prasad M K, Sinha A, and Chau Wang L-L 1980 Phys. Lett. B 91387 